\title{
Mécanismes physiologiques et neurochimiques impliqués dans le bruxisme du sommeil
}

\author{
Physiological and neurochemical mecanisms involved in sleep bruxism
}

\author{
OMARJEE REYHANA ${ }^{1}$, DIDIER CUGY ${ }^{2}$, YVES DELBOS ${ }^{3}$
}

\section{RÉSUMÉ}

Bien que l'aspect multifactoriel du bruxisme ne soit pas remis en cause, l'hypothèse du rôle des neuromédiateurs du système nerveux central dans l'initiation du bruxisme est actuellement explorée. Lorsqu'il se manifeste au cours du sommeil, le bruxisme est associé au phénomène d'éveil et correspond à une activité rythmique des muscles masticateurs se manifestant par des bruits occlusaux de type grincements de dents et des activités accrues sur l'EEG, l'ECG et l'EMG. II peut coexister avec d'autres pathologies du sommeil et avoir des conséquences sur l'état général du patient. Le diagnostic du bruxisme du sommeil par l'odontologiste va s'appuyer, d'une part sur des éléments rapportés par le patient et d'autre part, sur des signes observés lors de l'examen clinique. Cela va permettre au praticien d'orienter le patient vers un spécialiste adéquat en cas de besoin. L'examen polysomnographique permet de préciser le type de bruxisme et les pathologies associées. La prise en charge de la parafonction comporte une approche psychocomportementale, la physiothérapie et le port d'un appareil orthopédique, complétés par des thérapeutiques restauratrices médicamenteuses, si nécessaire. Med Buccale Chir Buccale 2007; 13 : 129-138.

mots clés: bruxisme, sommeil, neurochimie, diagnostic, thérapeutiques

\section{SUMMARY}

Although the multifactorial aspect of the bruxism is not questioned, the path of the role of the neuromediators of the central nervous system in the initiation of the bruxism is investigated now. When it appears sleeping, bruxism associated to microarousal and corresponds to a activity of the muscles, appearing with grinding sounds and increased in EEG, ECG and EMG. be met with other sleep disorders and affect the patient's health. The diagnosis of sleep bruxism based on both reported and in this way, the patient may be directed to the specialist if it is necessary. Polysomnography is required to specify the characteristics of bruxism and related disorders. Managing sleep bruxism includes psychological and behavioral therapy, physiotherapy and orthopedic appliance. Some restorative treatments complete bruxism management if needed. Med Buccale Chir Buccale 2007; 13 : 129-138. key words: bruxism, sleep, neurochimestry, diagnosis, management

\footnotetext{
1. Faculté d'Odontologie Bordeaux

2. Service d'Exploration Fonctionnelle du Système Nerveux, CHU de Bordeaux

3. Pôle d'Odontologie et de Santé Buccale, CHU de Bordeaux
}

Demande de tirés à part:

Yves Delbos UFR d'Odontologie 16-20 cours de la Marne 33082 Bordeaux cedex yves.delbos@odonto.u-bordeaux2.fr 
médecine

buccale

chirurgie

buccale

VOL. $13, \mathrm{~N}^{\circ} 3$ 2007

page 130
Le bruxisme n'est pas un phénomène d'apparition récente, le « grincement de dents " a déjà été décrit dans la Bible (Matthieu, 8-12 ; 13-42 ; 25-30). Au début du XX siècle, Marie et Pietkiewiecz ${ }^{[16]}$ ont rapporté les premières observations cliniques liées à cette parafonction. Pourtant, après plus d'un siècle de réflexion, l'étiologie du bruxisme et sa physiopathologie font toujours l'objet de recherches... La piste du rôle des neuromédiateurs du système nerveux central dans l'initiation du bruxisme lié au sommeil est actuellement privilégiée, bien que l'aspect multifactoriel (stress, drogues, médicaments...) reste généralement admis.

Cet article aura pour but de décrire les caractéristiques propres au bruxisme du sommeil, qui est à la fois une parafonction et une parasomnie. Nous présenterons ensuite les théories les plus récentes les mécanismes d'apparition du bruxisme au cours du sommeil, mettant en jeu les neuromédiateurs impliqués dans la mastication d'une part, et dans le sommeil d'autre part.

\section{BRUXISME ET SOMMEIL}

\section{Le sommeil physiologique chez l'homme}

Le sommeil est un besoin physiologique et fondamental qui occupe le tiers de l'existence de l'être humain [31]. Il est constitué de 4 à 5 cycles selon la durée du sommeil (Fig. 1), alternant deux phases très distinctes : le sommeil lent (léger ou profond) et le sommeil paradoxal [2]. Les deux ou trois premiers cycles comportent du sommeil lent et profond alors que les derniers cycles présentent des épisodes de sommeil paradoxal de plus longue durée mais ce schéma reste variable selon l'âge du sujet [2]. Chaque cycle dure environ 90 minutes et on note la présence de brefs éveils à la fin des cycles dont le sujet n'a, la plupart du temps, aucun souvenir [30].

L'étude approfondie des différents stades du cycle veille-sommeil est réalisée par la polysomnographie : c'est une technique qui associe l'enregistrement de l'activité électroencéphalographique (EEG) engendrée par des générateurs cérébraux, de l'activité électrique dépendant des mouvements des globes oculaires (EOG) et de l'activité électrique des muscles du menton (EMG) ${ }^{[4]}$. On distingue [22] :

- La veille qui se compose de la veille active et de la veille calme. La veille active correspond à une activité EEG rapide et de bas voltage et à des mouvements des yeux et des paupières. La veille calme, au repos, les yeux clos, présente une activité EEG caractérisée par des ondes alpha ainsi qu'une absence de mouvements oculaires.

- Le sommeil lent qui comprend quatre stades de profondeur croissante : le stade 1, ou stade d'endormissement ; le stade 2, dit sommeil lent léger ; les stades 3 et 4 qui constituent le sommeil lent profond. Pendant tous ces stades, le tonus musculaire demeure présent, même si son amplitude

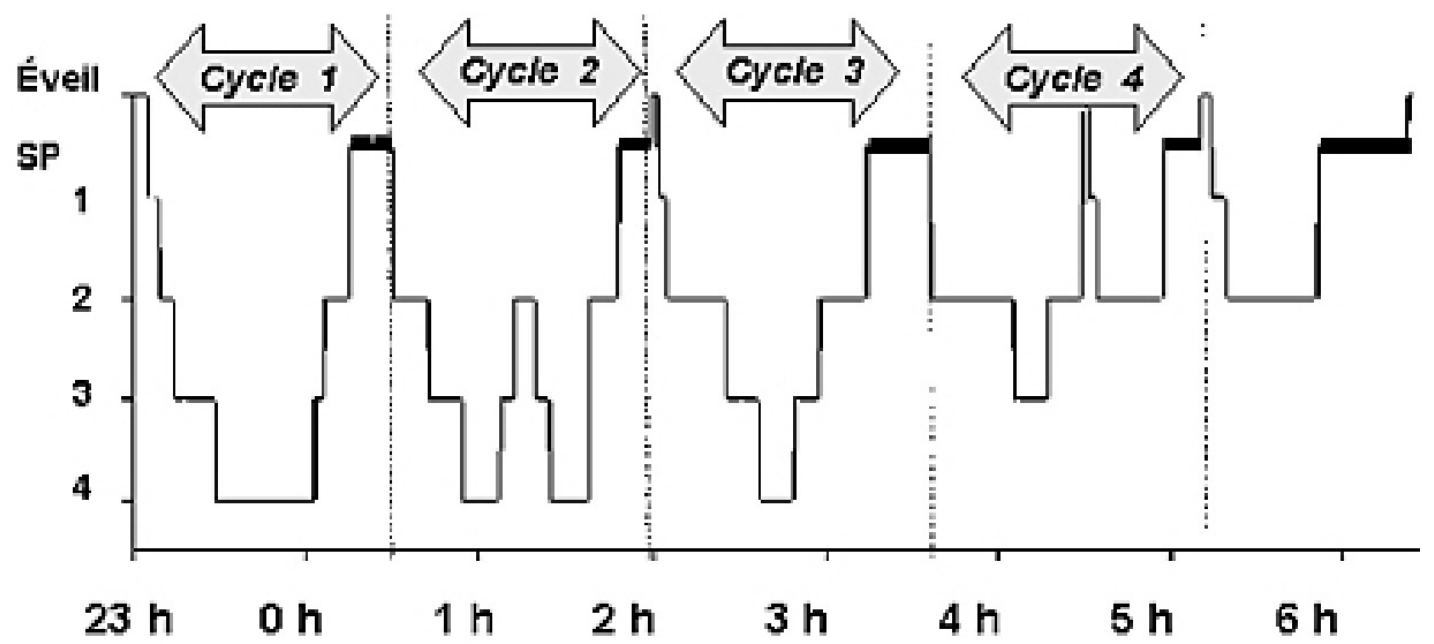

Figure 1 : Hypnogramme d'une nuit complète chez un adulte jeune. Hypnogram of a young adult complete night. 
tend à diminuer dans les stades les plus profonds. L'EOG ne montre aucun mouvement oculaire : on parle de phase non-REM (non rapid eye movement) du sommeil.

- Le sommeil paradoxal qui est ainsi appelé car il associe des signes du sommeil profond et des signes de l'éveil [30]. Des mouvements oculaires apparaissent, isolés ou en bouffées, sous les paupières closes. On parle de stade REM (rapid eye movement) du sommeil. Le tonus musculaire est aboli, à l'exception de brèves décharges musculaires ou twitches affectant les muscles du visage et des extrémités [2].

La régulation du cycle veille-sommeil (rythme circadien) fait intervenir cinq éléments : le réseau du sommeil lent situé dans le cerveau antérieur, le réseau du sommeil paradoxal constitué de plusieurs groupes de neurones particuliers, le système d'éveil, le système anti-éveil et l'horloge biologique. Les pacemakers du sommeil lent et du sommeil paradoxal sont sous le contrôle inhibiteur de l'éveil, lui-même entretenu par les stimulations de l'environnement et du milieu interne. L'endormissement est le résultat du blocage de l'éveil à un moment donné du nycthémère par un système anti-éveil situé dans l'hypothalamus et mis en route par une des composantes de l'éveil, la sérotonine. Dans le réseau de l'éveil, l'information circule entre les neurones grâce à des neurotransmetteurs comme l'acétylcholine, l'adrénaline, la noradrénaline, I'histamine et la sérotonine (5-HT). Ces neurones de l'éveil vont diminuer ou arrêter leur activité pendant le sommeil [2, 32, 33]. Les systèmes GABAergique et glycinergique prennent alors le relais ; ce sont des systèmes inhibiteurs qui sont responsables, entre autres, de l'atonie musculaire durant le sommeil [2].

Par ailleurs, l'alternance sommeil lent-sommeil paradoxal (rythme ultradien) semble avoir un support métabolique. En effet, au cours du sommeil paradoxal, le cerveau consomme autant de glucose et d'oxygène que pendant l'éveil, alors que durant le sommeil lent, il y a économie d'énergie et reconstitution des réserves énergétiques. Ainsi, la survenue et la durée de la phase REM du sommeil vont être directement dépendantes des réserves énergétiques disponibles.

\section{Le bruxisme : manifestation exagérée d'une activité normalement observée au cours du sommeil}

L'activité rythmique des muscles masticateurs se retrouve chez $60 \%$ de la population. Cependant, elle est trois fois plus importante en terme de fréquence chez les sujets bruxistes (en moyenne 5,4 épisodes par heure contre 1,6 chez les sujets asymptomatiques) et présente une amplitude plus grande que les sujets non-bruxistes ${ }^{[1,8,31]}$. Pour Lavigne et coll., cette activité rythmique des muscles masticateurs est huit fois plus fréquente chez les bruxistes [12].

Il existe deux types d'activité rythmique des muscles masticateurs (ou ARMM) durant le sommeil [10] :

- La première se caractérise par des bouffées répétitives des muscles de fermeture et une activité électromyographique basse. Elle concerne $56 \%$ de la population et ne constitue pas une manifestation de bruxisme.

- La seconde montre une activité large et phasique des muscles élévateurs et diducteurs résultant de contractions musculaires de grande amplitude, et une activité tonique des muscles élévateurs résultant de mouvements restreints mais très soutenus. Elle concerne $6 \%$ de la population et correspond à la véritable manifestation du bruxisme : on a une fréquence élevée des épisodes d'activité musculaire tonique et phasique [13]. Les contacts dentaires involontaires observés au cours de la nuit restent des évènements tout à fait normaux lorsqu'ils sont associés à la déglutition réflexe rencontrée au cours du sommeil $[8,25,31]$. Cependant, la durée et l'intensité de ces contacts varient entre les sujets asymptomatiques et les sujets bruxistes, ce qui fait de ces contacts des évènements pathologiques chez ces derniers. Selon Kydd et Daly, la durée des contacts dentaires au cours du sommeil est supérieure de 15 à 45 minutes chez les sujets bruxistes. Ceux-ci présentent une durée moyenne de 11,4 minutes de contacts dentaires contre 3,1 minutes chez les sujets non bruxistes. C'est une différence nette et statistiquement significative ${ }^{[9]}$. De plus, l'intensité de la force occlusale exercée au cours d'un épisode de bruxisme du sommeil peut parfois excéder l'intensité maximale de serrage obtenue volontairement durant la journée [21]. médecine

buccale

chirurgie

buccale

VOL. $13, \mathrm{~N}^{\circ} 3$ 2007

page 131 
médecine

buccale

chirurgie

buccale

VOL. $13, \mathrm{~N}^{\circ} 3$ 2007

page 132
En 2003, Miyawaki et coll. ont étudié l'influence de la position de sommeil sur le bruxisme et les réflexes de déglutition. Ils ont constaté que les sujets bruxistes passent 95,5\% de leur temps de sommeil dans la position couchée sur le dos ou en décubitus latéral. De plus, chez ces sujets, $74 \%$ des épisodes d'ARMM et de déglutition ont lieu lorsque les sujets sont allongés sur le dos (contre $23 \%$ en position latérale) ${ }^{[20]}$. Dans une seconde étude réalisée en 2004, Miyawaki et coll. ont montré que $88,1 \%$ des épisodes de ARMM ont lieu en position de sommeil sur le dos [19]. Ainsi, dormir sur le dos favoriserait l'activité rythmique des muscles masticateurs.

\section{Les conditions d'apparition du bruxisme du sommeil}

L'organisation du sommeil chez le sujet bruxiste apparaît généralement normale en terme de durée, de répartition des différents stades et d'efficacité ${ }^{[5,11]}$. Les sujets jeunes, souffrant de bruxisme nocturne, présentent une macrostructure normale du sommeil et ne se plaignent pas de troubles du sommeil. En revanche, on note une diminution de la qualité du sommeil chez les patients âgés ou ceux qui souffrent d'apnée du sommeil, par exemple ${ }^{[7,8]}$.

De nombreux travaux ont montré que le bruxisme peut apparaître pendant toutes les phases du sommeil, mais de façon prédominante dans le stade 2, soit au cours du sommeil lent léger [10, 27, 32]. Au cours d'études, les auteurs ont pu remarquer que des changements d'ondes cérébrales apparaissaient avant un épisode de bruxisme, témoignant d'une activité corticale accrue [27, 28, 29]. Par ailleurs, Clarke et Townsend ont étudié la distribution des épisodes de bruxisme chez une dizaine de patients présentant cette parasomnie. Ils notent que cette répartition pourrait correspondre au rythme ultradien du sommeil, avec une récurrence toutes les 90 à 100 minutes en moyenne [13]. La nature cyclique des épisodes de bruxisme au cours du sommeil est cohérente et vient soutenir la théorie d'une étiologie d'origine centrale. Kato et coll. ont noté que la fréquence de l'ARMM est plus élevée chez les sujets bruxistes et qu'elle se manifeste après des micro-éveils spontanés transitoires. Ils ont entrepris alors une expérience qui consiste à provoquer des micro-éveils chez des sujets bruxistes et non-bruxistes. Ils ont observé une activité des muscles masséters chez tous les patients bruxistes après un micro-éveil provoqué, contre un seul sujet du groupe témoin. Ainsi, ces auteurs émettent l'hypothèse que le bruxisme du sommeil serait une activité orofaciale exagérée, associée aux micro-éveils nocturnes [6]. Il est important de noter que les sujets bruxistes présentent un nombre de micro-éveils durant une nuit de sommeil qui est tout à fait normal, ce qui est en accord avec le fait que la macrostructure de leur sommeil n'est pas perturbée [12]. On parle, bien évidemment, de cas de bruxisme pur, c'està-dire sans manifestations pathologiques concomitantes. En effet, comme l'a souligné Monteyrol lors de la conférence sur le syndrome d'apnée obstructive du sommeil (SAOS) organisée par l'ONFOC le 17 novembre 2005 à Bordeaux, lorsqu'un sujet souffre d'un SAOS concomitant au bruxisme, le nombre de micro-éveils nocturnes est augmenté car l'épisode d'apnée nécessite un effort thoracique pour contrer l'obstacle et permettre la ventilation du patient. Cet effort musculaire est possible grâce à un éveil partiel du sujet. Ainsi, si un patient présente un bruxisme concomitant à un SAOS, la macrostructure de son sommeil est perturbée et présente de nombreux micro-éveils. En plus des changements de l'activité corticale déjà décrits, les sujets souffrant de bruxisme du sommeil vont présenter des modifications de l'activité autonome, se traduisant par une tachycardie, phénomène transitoire qui précède et accompagne l'épisode de bruxisme, une vasoconstriction du bout des doigts et des changements de potentiels de la paume et de l'avantbras (normalement nombreux à l'état d'éveil). L'hypnogramme (Fig. 2) montre précisément les modifications de type autonome et électrocorticales décrites précédemment. Ce tracé a été réalisé par le Dr. Didier Cugy, médecin spécialisé dans la pathologie du sommeil et de la vigilance à Bordeaux.

\section{Manifestations accompagnant le bruxisme du sommeil}

Le bruxisme du sommeil peut être associé à d'autres manifestations motrices survenant pen- 


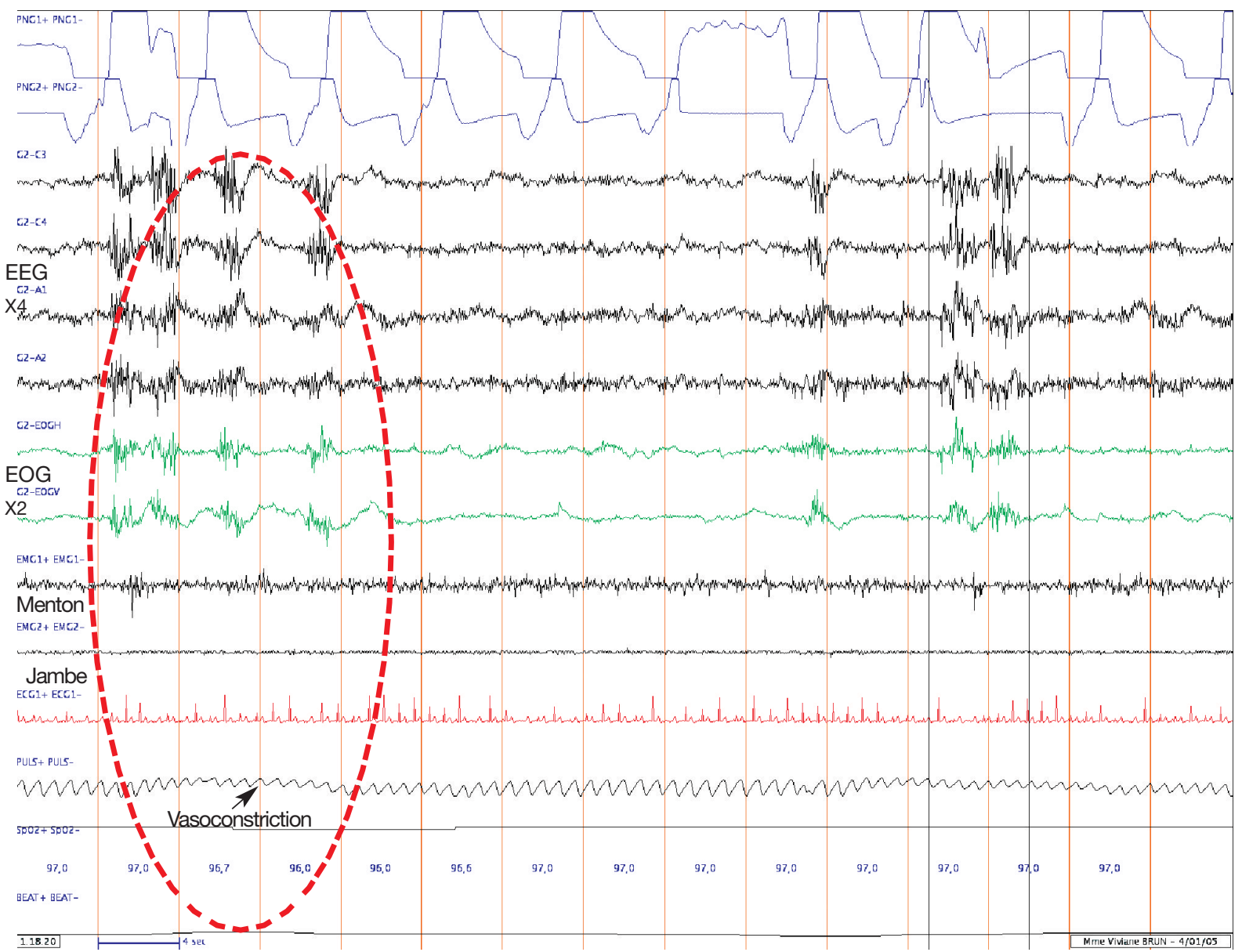

médecine

buccale

chirurgie

buccale

VOL. $13, N^{\circ} 3$

2007

page 133

Figure 2 : Tracé polysomnographique montrant un épisode de bruxisme au cours du sommeil avec les changements électrocorticaux et végétatifs qui l'accompagnent.

L'apparition du bruxisme au cours du sommeil va donc suivre trois étapes [8, 11] :

- Tout d'abord, des changements de l'activité électro-corticale vont précéder l'épisode de bruxisme de 4 secondes.

- Puis, une activation du rythme cardiaque apparaît 1 seconde avant le bruxisme.

- Enfin, l'activité rythmique des muscles masticateurs va engendrer le bruxisme et, dans $50 \%$ des cas, il y a une forte déglutition.

Polysomnographic graph showing an episode of bruxism during the sleep with the électrocortical and vegetative changes which accompany it.

The apparition of the bruxism during the sleep is thus going to follow three stages ${ }^{[8,11]}$ :

- First of all, changes of the electro-cortical activity are going to precede the episode bruxism of 4 seconds.

- Then, an activation of the cardiac rhythm appears 1 second before the bruxism.

- Finally, the rhythmic activity of the masticatory muscles is going to engender the bruxism and, in $50 \%$ of the cases, there is a strong gulp.

dant le sommeil, telles que des mouvements du corps, des mouvements périodiques du sommeil et des mouvements liés à des troubles respiratoires comme le ronflement ou l'apnée obstructive du sommeil [1]. Phillips et coll. ont d'ailleurs noté que l'apnée du sommeil provoque une perturbation de la structure du sommeil avec des micro-éveils fréquents [23]. Or, ces phénomènes nocturnes sont le terrain favori du développement de l'activité parafonctionnelle qu'est le bruxisme. Ils enregistrent en moyenne 12,2 épisodes de bruxisme centré par heure chez les patients souffrant d'apnée du sommeil contre 7,6 épisodes par heure chez les sujets témoins. Ils concluent à une 
médecine

buccale

chirurgie

buccale

VOL. $13, \mathrm{~N}^{\circ} 3$ 2007

page 134 corrélation significative entre les épisodes d'apnées du sommeil et le serrement/grincement des dents [24]. Kato et al. citent une étude épidémiologique, réalisée en 2001 par Ohayon et coll., qui montre que les troubles respiratoires du sommeil (et en particulier le SAOS) sont plus fréquents chez les sujets bruxistes (3,4 à 4,8\%) que chez ceux qui ne le sont pas $(1,4 \%)^{[8]}$.

Lavigne et coll. décrivent une augmentation du flux salivaire avant l'apparition de l'ARMM [13]. Thie et coll. pensent que cette salivation, provoquée par les mouvements orofaciaux, permettrait la lubrification des structures oro-œsophagiennes, assurant ainsi leur santé et leur intégrité [30]. Enfin, Miyawaki et coll. montrent que $60 \%$ des ARMM sont associés à un mouvement de déglutition. Ils précisent que ce mouvement est plus fréquent au cours du sommeil chez les patients bruxistes (6,8 épisodes par heure) que chez les sujets du groupe témoin (3,7 épisodes par heure) [20].

Ainsi, selon ces premiers éléments, le bruxisme du sommeil relèverait d'une activité rythmique des muscles masséters (ARMM) associée à des contacts dentaires, étroitement liés au réflexe de déglutition qui persiste au cours du sommeil. Cette activité, présente chez $60 \%$ de la population, serait exacerbée chez les sujets bruxistes, présentant une ARMM plus soutenue et des contacts dentaires plus importants en durée et en intensité. La position de sommeil serait, à priori, un facteur intervenant sur la fréquence de ces activités. De part les composantes corticales et autonomes qui accompagnent l'épisode de bruxisme, on associe le bruxisme au phénomène de microéveil : le bruxisme du sommeil serait une réaction d'éveil exagérée, accompagnée par des manifestations physiologiques (salivation, déglutition) et pathologiques (apnée, mouvements périodiques) diverses.

\section{THEORIES NEUROCHIMIQUES}

\section{La dopamine}

\section{La théorie dopaminergique}

Dans la pathogenèse du bruxisme, les mécanismes dopaminergiques ont été analysés pour diverses raisons : influence des psychostimulants dopaminergiques sur le bruxisme (Hartmann, 1994 ; Lavigne et coll., 1995 ; Kato et coll., 2001), apparition du bruxisme dans des maladies telles que la maladie de Parkinson impliquant la dopamine. Par ailleurs, le bruxisme a été défini comme un trouble du mouvement stéréotypé et périodique. Or, le rôle du système dopaminergique dans le contrôle des comportements stéréotypés et dans les troubles moteurs durant le sommeil est bien établi [10]. II semblerait que, selon les récepteurs et les circuits dopaminergiques mis en jeu, le neuromédiateur aurait un rôle modulateur : les récepteurs D1-like (D1 et D5) favoriseraient le bruxisme alors que les récepteurs $\mathrm{D} 2$-like (D2 et D4) auraient un effet contraire. En effet, une expérience citée par Chapotat et coll. ${ }^{[4]}$ montre que l'administration directe des agonistes des récepteurs $\mathrm{D} 1$ dans les ganglions de la base entraîne une augmentation des mouvements oromandibulaires chez le rat et le chat, alors que celle des agonistes des récepteurs D2 les diminuent (Johansson et coll., 1987 ; Koshikawa et coll. 1990 ; Spooren et coll. 1991) [10]. De même, une forte dose de L-Dopa favorise la parafonction tandis qu'une faible dose a un effet bénéfique. Ce phénomène est compatible avec d'autres agonistes dopaminergiques qui possèdent une action bidirectionnelle [10]. En 1997, Lobbezoo et coll. montrent que, après deux semaines d'administration de bromocriptine (agoniste des récepteurs D2-like) chez deux patients, ceux-ci présentent une diminution des épisodes de bruxisme nocturne de 20 à $30 \%$ [15]. Lavigne et coll. trouvent, au contraire, que la bromocriptine ne présente aucun effet chez les patients bruxistes [14].

\section{La relation entre la dopamine et les fac- teurs connus pour favoriser le bruxisme et le sommeil}

La dopamine joue un rôle dans la motricité, la schizophrénie, la maladie de Parkinson et se retrouve aussi dans l'œil et dans le réseau neuronal à partir du nez. De plus, il s'agit d'un neurotransmetteur important entre le cerveau et l'hypophyse, une glande qui produit des hormones importantes pour la croissance, le développement sexuel et d'autres fonctions corporelles. En cas d'anxiété, de grandes quantités de dopamine sont produites, 
entraînant une fixation sur la source de la crainte et préparent les muscles à une réaction rapide. En ce sens, la dopamine régule les effets plus sauvages des autres peptides stimulants comme l'adrénaline et la noradrénaline, en combinant vivacité et concentration. La vitamine B6 et le magnésium sont essentiels à la production de la dopamine, ce qui expliquerait qu'un déficit en magnésium puisse être un facteur étiologique du bruxisme. De même, fumer augmenterait les concentrations de dopamine, déséquilibrant le système dopaminergique et facilitant l'apparition du bruxisme. Concernant les états de vigilance, on admet d'une façon générale que la dopamine accroît la vigilance mais son rôle dans le sommeil est moins clair. Par ailleurs, il semble exister une atteinte du système dopaminergique au niveau du système nerveux central dans le syndrome des mouvements périodiques du sommeil.

\section{Les autres cathécolamines}

\section{Les études mettant en cause les autres cathécolamines dans la genèse du bruxisme}

Comme le soulignent Bader et Lavigne, la L-dopa n'étant pas un précurseur spécifique de la dopamine, il convient de se demander si les autres catécholamines, telles que l'adrénaline ou la noradrénaline, ne pourraient pas intervenir dans la régulation du bruxisme. Ainsi, ils rappellent que certains auteurs ont démontré que le propanolol, un béta-bloquant adrénergique, peut réduire le bruxisme du sommeil [1]. Récemment, Macaro et coll. ont tenté d'étudier les voies anatomiques pouvant exister entre le système dopaminergique et le noyau moteur du nerf trijumeau. Ils ont réussi à mettre en évidence des traces d'activité synaptique mettant en jeu des catécholamines, reliant le noyau moteur trigéminal à deux zones distinctes du cerveau [18] : I'aire prémotrice (ou aire psychomotrice), reliée au contrôle masticatoire, et des structures sous-corticales (amygdale et thalamus), liées au système nerveux autonome et aux réponses face au stress. Ainsi, ils apportent un argument anatomique à cette hypothèse dopaminergique ainsi qu'à l'étiologie psychologique et montrent que les catécholamines ont bien une action sur le contrôle neurophysiologique et neuropsychologique des mouvements orofaciaux. Enfin, un taux significatif de catécholamines a été retrouvé dans les urines de patients souffrant de bruxisme ${ }^{[1,18]}$.

\section{La relation entre les cathécolamines, le bruxisme et le sommeil}

L'adrénaline est à la fois une hormone médullosurrénale et un neurotransmetteur dans le système nerveux central. Elle fait partie du réflexe lutte/fuite. Elle accélère la vitesse de la respiration, dilate les pupilles et accroît le rythme cardiaque. Issu du tronc cérébral, le système adrénergique appartient aux circuits régulant le tonus sympathique et la température centrale inter-régulés avec les états de vigilance. La noradrénaline est également un neuromédiateur central et périphérique et une hormone sécrétée par la glande surrénale, surtout dans les situations de stress. Elle exerce un effet de grande excitation et, en comparaison avec l'adrénaline, elle entraîne plutôt de l'agressivité que de l'anxiété. Des drogues comme l'amphétamine et la cocaïne accroissent la quantité de noradrénaline, la première par la stimulation de sa production, la deuxième par l'inhibition de son catabolisme. Elles provoquent ainsi un état de vivacité, d'hyperactivité. Or, nous savons que de telles substances (amphétamine et cocaïne) provoquent des phénomènes de bruxisme chez les sujets étudiés. Une concentration trop élevée en noradrénaline est aussi retrouvée dans la tachycardie. Enfin, la noradrénaline intervient en synergie avec la dopamine et l'acétylcholine pour déterminer l'éveil, et s'oppose à l'action sédative d'autres neurotransmetteurs (GABA, 5-HT).

\section{Les mécanismes sérotoninergiques}

\section{Les théories avancées}

Certains antidépresseurs tels que les inhibiteurs sélectifs de la recapture de la sérotonine ou ISRS (par exemple le Prozac ${ }^{\circledR}$ ) sont connus pour exacerber le serrement et parfois le grincement des dents. En effet, I'ISRS provoquerait l'augmentation de la sérotonine extra-pyramidale, inhibant ainsi les voies dopaminergiques de contrôle des mouvements. Dans une étude décrivant des phé- médecine

buccale

chirurgie

buccale

VOL. $13, N^{\circ} 3$ 2007

page 135 
médecine

buccale

chirurgie

buccale

VOL. $13, \mathrm{~N}^{\circ} 3$ 2007

page 136 nomènes de bruxisme apparus chez quatre patients sous ISRS, Bostwick et Jaffee montrent que la prise de buspirone, agoniste de la sérotonine, entraîne la disparition des signes ${ }^{[3]}$. Par contre, l'utilisation d'un précurseur de la sérotonine (le tryptophane) ou d'un inhibiteur de sa réabsorption (l'amytriptyline) n'entraîne ni augmentation ni diminution du bruxisme ${ }^{[10,26]}$.

\section{Sérotonine, facteurs de risque $d u$ bruxisme et sommeil}

La sérotonine influence de très nombreuses régions du corps et de l'esprit. On notera en particulier ses effets comportementaux. Ainsi, via les amygdales, la sérotonine influence nos émotions de façon importante. Par ailleurs, la sérotonine fait partie des neuromédiateurs de l'éveil jouant également un rôle non négligeable dans l'endormissement. Une augmentation de la quantité de sérotonine est impliquée dans les troubles du sommeil.

\section{Histamine et bruxisme du sommeil}

Nous soulevons ici l'hypothèse selon laquelle I'histamine pourrait jouer un rôle dans les mécanismes d'apparition du bruxisme du sommeil pour deux raisons :

- Un lien a été établi entre les enfants allergiques et les phénomènes de bruxisme du sommeil. Or, le rôle de l'histamine dans les phénomènes allergiques est bien établi [17].

- Par ailleurs, l'étude du sommeil nous a montré que ce neuromédiateur joue un rôle dans les états de vigilance et fait partie des neuromédiateurs de l'éveil. Or, le bruxisme du sommeil est associé à des épisodes de micro-éveils durant la nuit ${ }^{[4,6]}$. Pour ces raisons, il semble très probable que le système histaminergique soit également impliquée, au même titre que les autres amines biogènes cités plus haut.

Outre son rôle dans les réactions d'hypersensibilité, l'histamine possède de nombreuses propriétés : relâchement des petites artères, contraction des muscles de l'intestin et des bronches, sécrétion d'acide chlorhydrique dans le suc gastrique, accélération du cœur, relâchement des contractions de l'utérus et joue un rôle important dans les mécanismes de l'inflammation, de l'anaphylaxie et dans les réactions d'intolérance alimentaire et d'allergie. Enfin, on a vu que l'histamine est un des neuromédiateurs qui participent au système de l'éveil [32, 33]. Les neurones qui la synthétisent sont actifs durant l'éveil, et la pharmacologie de l'histamine soutient son rôle important dans l'éveil et permet d'expliquer la somnolence induite par les antihistaminiques ${ }^{[23] .}$

\section{SYNTHÈSE}

Le Tableau 1 montre les effets parallèles de ces neuromédiateurs qui vont, d'une part, faciliter les mouvements masticateurs, et d'autre part, promouvoir les phénomènes d'éveil et de micro-éveil associé au bruxisme au cours du sommeil [10]. Les études concernant la neurophysiopathologie du bruxisme sont très nombreuses mais elles ne permettent pas de conclure sur une explication neurochimique unique et simple. II semble aujourd'hui que le rôle attribué à ces neurotransmetteurs est beaucoup moins clair qu'on le prétendait originalement, comme le souligne Lavigne et coll. [10]. La littérature comporte de nombreuses controverses et elle est essentiellement basée sur des rapports de cas anecdotiques. Cependant, nous avons vu que les amines biogènes (catécholamines et histamine) agissent, de façon physiologique, sur la motricité (la dopamine en particulier), les émotions (anxiété, agressivité, réflexe lutte/fuite), le système sympathique (accélération du rythme cardiaque, de la respiration...) et les mécanismes d'éveil. Or, le bruxisme du sommeil est considéré comme un phénomène moteur stéréotypé, survenant lors de phénomènes de microéveils nocturnes, accompagné de manifestations végétatives (tachycardie...) et dont l'étiologie multifactorielle, à la fois périphérique et centrale, semble être largement soumise à des facteurs psychogènes non négligeables. Ainsi, on constate que tous les neuromédiateurs incriminés au cours des différentes études dans la genèse du bruxisme sont effectivement liés à des manifestations générant ou accompagnant la parafonction. II paraît difficile de conclure sur une implication neurochimique unique et simple mais il est évident que toutes ces substances ont un rôle à jouer dans la physiopathologie du bruxisme du sommeil. 
Tableau 1 : Rôle des amines biogènes dans la genèse de la mastication ainsi que dans le contrôle moteur et le contrôle de la vigilance au cours du sommeil (d'après Lavigne et coll. ${ }^{[10]}$ ).

Role of biogene amines in the chewing genesis as well as in the motor control and the control of the vigilance during the sleep (according to Lavigne and coll. ${ }^{[10]}$ ).

\begin{tabular}{|c|c|c|}
\hline & Mastication & Sommeil \\
\hline Dopamine & $\begin{array}{l}\text { Favorise le mouvement rythmique des } \\
\text { muscles masticateurs. }\end{array}$ & $\begin{array}{l}\text { Favorise l'état d'alerte et l'éveil. } \\
\text { Facteur majeur dans la physiopatho- } \\
\text { logie des mouvements périodiques } \\
\text { du sommeil. }\end{array}$ \\
\hline Adrénaline ou Noradrénaline & $\begin{array}{l}\text { Facilite l'activité rythmique des } \\
\text { muscles masticateurs induite par le } \\
\text { système activateur (glutamate). }\end{array}$ & Favorise les états d'alerte et d'éveil. \\
\hline Sérotonine & $\begin{array}{l}\text { Facilite l'activité rythmique des } \\
\text { muscles masticateurs. }\end{array}$ & $\begin{array}{l}\text { Favorise l'endormissement et dimi- } \\
\text { nue son activité au cours du sommeil } \\
\text { non-REM et REM. }\end{array}$ \\
\hline Histamine & $\begin{array}{l}\text { Facilite l'activité rythmique des } \\
\text { muscles masticateurs. }\end{array}$ & Favorise les états d'alerte et d'éveil. \\
\hline
\end{tabular}

\section{CONCLUSION}

Le bruxisme du sommeil apparait dans des conditions particulières faisant appel aux neuromédiateurs classiques impliqués dans la genèse de la mastication, du sommeil et dans le contrôle émotionnel et végétatif. De plus, il engendre des modifications corticales et autonomes non négligeables. Ainsi, le bruxisme est une réponse excessive du cerveau et du cœur associée à un éveil bref et inconscient du cerveau lors du sommeil. Le plus souvent, le patient n'a pas conscience de sa parasomnie et seul le bruit produit par le grincement des dents peut venir perturber le sommeil de son conjoint. II est probable que ces activités invoIontaires permettent de rétablir une lubrification des voies oro-oesophagiennes car elles s'accompagnent souvent d'une forte déglutition. Cependant, il arrive que cette parasomnie soit associée à des manifestations pathologiques telles que le ronflement ou l'apnée du sommeil. Dans ce cas, le bruxisme apparaît comme un symptôme pouvant parfois révéler un problème sous-jacent plus important. C'est pourquoi, une prise en charge complète et multidisciplinaire du patient qui présente des signes de bruxisme est nécessaire. Cette prise en charge doit être rigoureuse et ne rien laisser au hasard.

\section{RÉFÉRENCES}

1 - Bader G, Lavigne G. Sleep bruxism; an overview of an oromandibular sleep movement disorder. Sleep Med Rev $2000 ; 4$ : 27-43.

2 - Billard M. Le sommeil. Le Cavalier Bleu, collection «Idées Reçues », Paris, 2002.

3 - Bostwick JM, Jaffee MS. Buspirone as an antidote to SSRI-induced bruxism in 4 cases. J Clin Psychiatry $1999 ; 60: 857-60$.
4 - Chapotat B, Lin JS, Robin O, Jouvet M. Bruxisme du sommeil : aspects fondamentaux et cliniques. J Parodontol Implantol Orale 1999 ; 18 : 277-89.

5 - Clarke NG, Townseng GC. Distribution of nocturnal bruxism patterns in man. J Oral Rehabil 1984 ; 11 : 529-34.

6 - Kato T, Montplaisir JY, Guitard F, Sessile BJ, Lund JP, Lavigne GJ. Evidence taht experimentally induced sleep bruxism is a consequence of transient arousal. J Dent Res 2003 ; 82 : 284-8. médecine

buccale chirurgie buccale

VOL. $13, \mathrm{~N}^{\circ} 3$ 2007

page 137 
7 - Kato T, Thie NMR, Huynh N, Miyawaki S, Lavigne GJ. Topical review: Sleep Bruxism and the Role of Peripheral Sensory Influences. J Orofac Pain 2003 ; 17 : 191-206.

8 - Kato T, Thie NMR, Montplaisir JY, Lavigne GJ. Bruxism and orofacial movements during sleep. Dent Clin North Am 2001; 45 : 657-84.

9 - Kydd WL, Daly C. Duration of nocturnal tooth contacts during bruxing. J Prosthet Dent 1985 ; 53 : 717-21.

10 - Lavigne GJ, Kato T, Kolta A, Sessile BJ. Neurobilogical mechanisms involved in sleep bruxism. Crit Rev Oral Biol Med $2003 ; 14: 30-46$.

11 - Lavigne G. La genèse du bruxisme survenant lors du sommeil. Carrefour Dentaire, $2002 ; 2$ : 1. http://www. medent.umontreal.ca/medent/dentistes/chroniques/carrefour v3 web.pdf

12 - Lavigne GJ, Rompre PH, Guitard F, Sessile BJ, Kato T, Montplaisir JY. Lower number of K-complexes and Kalphas in sleep bruxism: a controled quantitative study. Clin Neurophysiol 2002 ; 113 : 686-93.

13 - Lavigne GJ, Rompre PH, Poirier G, Huard H, Kato T, Montplaisir JY. Rythmic masticatory muscle activity during sleep in humans. J Dent Res 2001 ; 80 : 443-8.

14 - Lavigne GJ, Soucy JP, Lobbezoo F, Manzini C, Blanchet PJ, Montplaisir JY. Double-blind, crossover, placebo-controlled trial of bromocriptine in patients with sleep bruxism. Clin Neuropharmacol 2001 ; 24 : 145-9.

15 - Lobbezoo F, Soucy JP, Hartman NG, Lavigne GJ. Effects of the D2 receptor agonist bromocriptine on sleep bruxism: report of two single-patient clinical trials. J Dent Res 1997 ; 76 : 1610-4.

16 - Marie MM, Pietkiewicz M Fils. La bruxomanie. Rev Stomatol $1907 ; 14: 107-16$.

17 - Marks MB. Bruxism in allergic children. Am J Orthod $1980 ; 77: 48-59$.

18 - Mascaro MB, Bittencourt JC, Casatti C, ELIAS CF. Alternative pathways for cethecolamine action in oral motor control. Neurosci Lett 2005 ; 386 : 34-9.

19 - Miyawaki S, Tanimoto Y, Araki Y, Katayama A, Imai M, Takano-Yamamoto T. Relationships among nocturnal jaw muscles activities, decrease esophageal $\mathrm{pH}$, and sleep positions. Am J Orthod Dentofac Orthoped 2004 ; $126: 615-9$.

20 - Miyawaki S, Lavigne GJ, Pierre M, Guitard F, Montplaisir JY, Kato T. Association between sleep bruxism, swallowing-related laryngeal movement, and sleep positions. Sleep $2003 ; 26$ : 461-5.

21 - Nishigawa K, Bando E, Nakano M. Quantitative study of bite force during sleep associated bruxism. J Oral Rehabil $2001 ; 28: 485-91$.

22 - Onen SH, Onen F. Dictionnaire de Médecine du Sommeil. Ellipses, Paris, 1998.

23 - Phillips BA, Okeson J, Paesani D, Gilmore R. Effects of sleep position on slep apnea and parafunctional activity. Chest 1986 ; $90: 424-9$.

24 - Powell RN, Zander HA. The frequency and distribution of tooth contact during sleep. J Dent Res 1965 ; 44 : 713-7.

25 - Raigrodski A, Christensen L, Mohamed S, Gardiner D. The effect of 4-week administration of amitriptyline on sleep bruxism. A double-blind crossever clinical study. J Craniomandib Pract $2001 ; 19$ : 21-5.

26 - Reding GR, Zepelin H, Robinson JE, Zimmerman SO, Smith VH. Nocturnal teeth-grinding: all nignt psychophysiologic studies. J Dent Res 1968 ; 47: 786-97.

27 - Satoh T, Haraka Y. Electrophysiological study on toothgrinding during sleep. Encephalogr Clin Neurophysiol 1973 ; 35 : 267-75.

28 - Tani K, Yoshii N, Yoshino I, Kobayashi E. Electroencephalographic study of parasomnia: sleeptalking, enuseris and bruxism. Physiol Behav 1966 ; 1 : 241-3.

29 - Thie NMR, Kato T, Bader G, Montplaisir JY, Lavigne GJ. The significance of saliva during sleep and the relevance of oromotor movements. Sleep Med Rev 2002 ; 6 : 21327.

30 - Valatx JL. La physiologie du sommeil. http://sommeil.univ-lyon1.fr/articles/cfes/sante/physio.html

31 - Wieselmann G, Permann R, Korner E, Flooh E, Reinhart $\mathrm{B}$, Moser F, Lechner H. Distribution of muscle activity during sleep in bruxism. Eur Neurol 1986 ; 25 Suppl 2 : 111-6.

32 - Les molécules d'information dans notre corps. http://www.bomi-1-sante.com/Lichaam en geest/serotonine peptiden dopamine adrenaline.htm (page consultée le 06/07/2007).

33 - Histamine. http://www.esculape.com/biologie/histamine.html (page consultée le 06/07/2007). 